\title{
On the Stability of Generalized Additive Functional Inequalities in Banach Spaces
}

\author{
Jung Rye Lee, ${ }^{1}$ Choonkil Park, ${ }^{2}$ and Dong Yun Shin ${ }^{3}$ \\ ${ }^{1}$ Department of Mathematics, Daejin University, Kyeonggi 487-711, South Korea \\ ${ }^{2}$ Department of Mathematics, Hanyang University, Seoul 133-791, South Korea \\ ${ }^{3}$ Department of Mathematics, University of Seoul, Seoul 130-743, South Korea
}

Correspondence should be addressed to Choonkil Park, baak@hanyang.ac.kr

Received 18 February 2008; Accepted 2 May 2008

Recommended by Ram Verma

We study the following generalized additive functional inequality $\|a f(x)+b f(y)+c f(z)\| \leq$ $\|f(\alpha x+\beta y+\gamma z)\|$, associated with linear mappings in Banach spaces. Moreover, we prove the Hyers-Ulam-Rassias stability of the above generalized additive functional inequality, associated with linear mappings in Banach spaces.

Copyright ( 12008 Jung Rye Lee et al. This is an open access article distributed under the Creative Commons Attribution License, which permits unrestricted use, distribution, and reproduction in any medium, provided the original work is properly cited.

\section{Introduction and preliminaries}

The stability problem of functional equations originated from a question of Ulam [1] concerning the stability of group homomorphisms. Hyers [2] gave a first affirmative partial answer to the question of Ulam for Banach spaces. Hyers' theorem was generalized by Aoki [3] for additive mappings and by Rassias [4] for linear mappings by considering an unbounded Cauchy difference. A generalization of the Rassias theorem was obtained by Găvruţa [5] by replacing the unbounded Cauchy difference by a general control function in the spirit of Rassias' approach.

Rassias [6] during the 27th International Symposium on Functional Equations asked the question whether such a theorem can also be proved for $p \geq 1$. Gajda [7] following the same approach as in Rassias [4] gave an affirmative solution to this question for $p>1$. It was shown by Gajda [7] as well as by Rassias and Šemrl [8] that one cannot prove Rassias' theorem when $p=1$. The counterexamples of Gajda [7] as well as of Rassias and Šemrl [8] have stimulated several mathematicians to create new definitions of approximately additive or approximately linear mappings (cf. Găvruţa [5], Jung [9] who among others studied the Hyers-Ulam stability of 
functional equations). The paper of Rassias [4] had great influence on the development of a generalization of the Hyers-Ulam stability concept. This new concept is known as Hyers-UlamRassias stability of functional equations (cf. the books of Czerwik [10], Hyers et al. [11]). During the last two decades, a number of papers and research monographs have been published on various generalizations and applications of the Hyers-Ulam-Rassias stability to a number of functional equations and mappings (see [12-17]).

Gilányi [18] showed that if $f$ satisfies the functional inequality

$$
\|2 f(x)+2 f(y)-f(x-y)\| \leq\|f(x+y)\|
$$

then $f$ satisfies the quadratic functional equation

$$
2 f(x)+2 f(y)=f(x+y)+f(x-y)
$$

see also [19]. Fechner [20] and Gilanyi [21] proved the Hyers-Ulam-Rassias stability of the functional inequality (1.1). Park et al. [22] investigated the Jordan-von Neumann-type CauchyJensen additive mappings and prove their stability, and Cho and Kim [23] proved the HyersUlam-Rassias stability of the Jordan-von Neumann-type Cauchy-Jensen additive mappings.

The purpose of this paper is to investigate the generalized additive functional inequality in Banach spaces and the Hyers-Ulam-Rassias stability of generalized additive functional inequalities associated with linear mappings in Banach spaces.

Throughout this paper, we assume that $X, Y$ are Banach spaces and that $a, b, c, \alpha, \beta, \gamma$ are nonzero complex numbers.

\section{Generalized additive functional inequalities}

Consider a mapping $f: X \rightarrow Y$ satisfying the following functional inequality:

$$
\|a f(x)+b f(y)+c f(z)\| \leq\|f(\alpha x+\beta y+\gamma z)\|
$$

for all $x, y, z \in X$.

We investigate the generalized additive functional inequality in Banach spaces.

We will use that for an additive mapping $f$, we have $f((m / n) x)=(m / n) f(x)$ for any positive integers $n, m$ and all $x \in X$ and so $f(r x)=r f(x)$ for any rational number $r$ and all $x \in X$.

Theorem 2.1. Let $f: X \rightarrow Y$ be a nonzero mapping satisfying $f(0)=0$ and (2.1). Then the following hold:

(a) $f$ is additive;

(b) if $\alpha / \beta, \beta / \gamma$ are rational numbers, then $a / \alpha=b / \beta=c / \gamma$;

(c) if $\alpha$ is a rational number, then $|a| \leq|\alpha|$.

Proof. (a) Letting $y=-(\alpha / \beta) x, z=0$ in (2.1), we get $a f(x)+b f(-(\alpha / \beta) x)=0$.

Letting $y=0, z=-(\alpha / \gamma) x$ in (2.1), we get $a f(x)+c f(-(\alpha / \gamma) x)=0$.

Letting $x=0, y=(\alpha / \beta) x, z=-(\alpha / \gamma) x$ in $(2.1)$, we get $b f((\alpha / \beta) x)+c f(-(\alpha / \gamma) x)=0$. 
Jung Rye Lee et al.

Thus, we get $f(-(\alpha / \beta) x)=-f((\alpha / \beta) x)$ and so $f(-x)=-f(x), b f(x)=a f((\beta / \alpha) x)$, and

$$
\frac{b}{a} f\left(\frac{\alpha}{\beta} x\right)=\frac{c}{b} f\left(\frac{\beta}{\gamma} x\right)=\frac{a}{c} f\left(\frac{\gamma}{\alpha} x\right)=f(x)
$$

for all $x \in X$.

On the other hand, letting $z=-(\alpha x+\beta y) / \gamma=-(\alpha / \gamma)(x+(\beta / \alpha) y)$ in (2.1), we get

$$
a f(x)+b f(y)+c f\left(-\frac{\alpha}{\gamma}\left(x+\frac{\beta}{\alpha} y\right)\right)=0 \text {. }
$$

The facts that

$$
c f\left(-\frac{\alpha}{\gamma}\left(x+\frac{\beta}{\alpha} y\right)\right)=c\left(-\frac{a}{c}\right) f\left(x+\frac{\beta}{\alpha} y\right)=-a f\left(x+\frac{\beta}{\alpha} y\right)
$$

and $b f(y)=a f((\beta / \alpha) y)$ give that

$$
f\left(x+\frac{\beta}{\alpha} y\right)=f(x)+f\left(\frac{\beta}{\alpha} y\right)
$$

and so $f(x+y)=f(x)+f(y)$ for all $x, y \in X$, which implies that $f$ is additive.

(b) Since $f$ is additive by (a) and since $\alpha / \beta$ and $\beta / \gamma$ are rational numbers, the facts that $(b / a) f((\alpha / \beta) x)=f(x)$ and $(c / b) f((\beta / \gamma) x)=f(x)$ give that

$$
\frac{b}{a} \cdot \frac{\alpha}{\beta} f(x)=\frac{c}{b} \cdot \frac{\beta}{\gamma} f(x)=f(x)
$$

for all $x \in X$. Since $f$ is nonzero, we conclude that $a / \alpha=b / \beta=c / \gamma$.

(c) Letting $y=z=0$ in (2.1), since $\alpha$ is a rational number, we get

$$
\|a f(x)\| \leq\|f(\alpha x)\|=\|\alpha f(x)\|
$$

for all $x \in X$. Since $f$ is nonzero, we conclude that $|a| \leq|\alpha|$, as desired.

As an application of Theorem 2.1, if we consider a mapping $f: X \rightarrow Y$ satisfying

$$
\|f(x)+f(y)+f(z)\| \leq\|f(x+2 y+3 z)\|
$$

for all $x, y, z \in X$, then we conclude that $f \equiv 0$.

Actually, for a mapping $f: X \rightarrow Y$ satisfying $f(0)=0$ and

$$
\|a f(x)+b f(y)+c f(z)\| \leq\|f(\alpha x+\beta y+\gamma z)\|
$$

for all $x, y, z \in X$, when $\alpha / \beta, \beta / \gamma$ are rational numbers, the above theorem says that $f \equiv 0$ unless $a / \alpha=b / \beta=c / \gamma$.

Here, we consider functional inequalities similar to (2.1). 
Remark 2.2. Let $f: X \rightarrow Y$ be a mapping with $f(0)=0$. If $f$ satisfies

$$
\|a f(x)+b f(y)+c f(z)\| \leq\|f(\alpha x+\beta y)\|
$$

for all $x, y, z \in X$, then by letting $x=y=0$, we get $c f(z)=0$ for all $z \in X$ and so $f \equiv 0$. And if $f$ satisfies

$$
\|a f(x)+b f(y)\| \leq\|f(\alpha x+\beta y+\gamma z)\|
$$

for all $x, y, z \in X$, then by letting $y=0, z=-\alpha x / \gamma$, we get $a f(x)=0$ for all $x \in X$ and so $f \equiv 0$.

In order to generalize the inequality (2.1), in the following corollaries, we assume that $a_{k}{ }^{\prime}$ s and $\alpha_{k}{ }^{\prime} s, k=1,2, \ldots, n(n \geq 3)$ are nonzero complex numbers.

Corollary 2.3. Let $f: X \rightarrow Y$ be a nonzero mapping satisfying $f(0)=0$ and

$$
\left\|\sum_{k=1}^{n} a_{k} f\left(x_{k}\right)\right\| \leq\left\|f\left(\sum_{k=1}^{n} \alpha_{k} x_{k}\right)\right\|
$$

for all $x_{k} \in X$. Then the following hold:

(a) $f$ is additive;

(b) if $\alpha_{j} / \alpha_{i}$ is a rational number, then $a_{i} / \alpha_{i}=a_{j} / \alpha_{j}$;

(c) if $\alpha_{i}$ is a rational number, then $\left|a_{i}\right| \leq\left|\alpha_{i}\right|$.

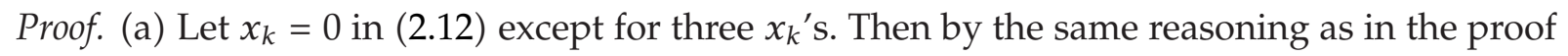
of Theorem 2.1, it is proved and so we omit the details.

(b) Letting $x_{i}=x, x_{j}=y$, by the same reasoning as in the corresponding part of the proof of Theorem 2.1, we can prove it.

(c) Letting $x_{k}=0$ for all $k$ with $k \neq i,(2.12)$ gives that

$$
\left\|a_{i} f\left(x_{i}\right)\right\| \leq\left\|f\left(\alpha_{i} x_{i}\right)\right\|=\left\|\alpha_{i} f\left(x_{i}\right)\right\| .
$$

Since $f$ is nonzero, we conclude that $\left|a_{i}\right| \leq\left|\alpha_{i}\right|$, as desired.

In the above corollary, similar to Remark 2.2, we notice that if a mapping $f$ satisfies $f(0)=0$ and

$$
\left\|\sum_{k=1}^{p} a_{k} f\left(x_{k}\right)\right\| \leq\left\|f\left(\sum_{k=1}^{q} \alpha_{k} x_{k}\right)\right\|
$$

for some $p, q \in\{1,2, \ldots, n\}$ with $p \neq q$ and all $x_{k} \in X$, then $f \equiv 0$.

Corollary 2.4. For an invertible $3 \times 3$ matrix $\left(a_{i j}\right)$ of complex numbers, let $f: X \rightarrow Y$ be a nonzero mapping satisfying $f(0)=0$ and

$$
\begin{aligned}
& \left\|a f\left(a_{11} x+a_{12} y+a_{13} z\right)+b f\left(a_{21} x+a_{22} y+a_{23} z\right)+c f\left(a_{31} x+a_{32} y+a_{33} z\right)\right\| \\
& \quad \leq\left\|f\left(\left(\alpha a_{11}+\beta a_{21}+\gamma a_{31}\right) x+\left(\alpha a_{12}+\beta a_{22}+\gamma a_{32}\right) y+\left(\alpha a_{13}+\beta a_{23}+\gamma a_{33}\right) z\right)\right\|
\end{aligned}
$$


Jung Rye Lee et al.

for all $x, y, z \in X$. Then the following hold:

(a) $f$ is additive;

(b) if $\alpha / \beta, \beta / \gamma$ are rational numbers, then $a / \alpha=b / \beta=c / \gamma$;

(c) if $\alpha$ is a rational number, then $|a|=|\alpha|$.

Proof. If we let $s=a_{11} x+a_{12} y+a_{13} z, t=a_{21} x+a_{22} y+a_{23} z, u=a_{31} x+a_{32} y+a_{33} z$, then since a matrix $\left(a_{i j}\right)$ is invertible and

$$
\left(\alpha a_{11}+\beta a_{21}+\gamma a_{31}\right) x+\left(\alpha a_{12}+\beta a_{22}+\gamma a_{32}\right) y+\left(\alpha a_{13}+\beta a_{23}+\gamma a_{33}\right) z=\alpha s+\beta t+\gamma u,
$$

inequality (2.15) is equivalent to

$$
\|a f(s)+b f(t)+c f(u)\| \leq\|f(\alpha s+\beta t+\gamma u)\|
$$

for all $s, t, u \in X$. Thus by applying Theorem 2.1, our proofs are clear.

By the same reasoning as in Remark 2.2, we obtain the following result.

Remark 2.5. For an invertible $3 \times 3$ matrix $\left(a_{i j}\right)$ of complex numbers, let $f: X \rightarrow Y$ be a mapping with $f(0)=0$. If $f$ satisfies

$$
\begin{aligned}
& \left\|a f\left(a_{11} x+a_{12} y+a_{13} z\right)+b f\left(a_{21} x+a_{22} y+a_{23} z\right)+c f\left(a_{31} x+a_{32} y+a_{33} z\right)\right\| \\
& \quad \leq\left\|f\left(\left(\alpha a_{11}+\beta a_{21}\right) x+\left(\alpha a_{12}+\beta a_{22}\right) y+\left(\alpha a_{13}+\beta a_{23}\right) z\right)\right\|
\end{aligned}
$$

or

$$
\begin{aligned}
& \left\|a f\left(a_{11} x+a_{12} y+a_{13} z\right)+b f\left(a_{21} x+a_{22} y+a_{23} z\right)\right\| \\
& \quad \leq\left\|f\left(\left(\alpha a_{11}+\beta a_{21}+\gamma a_{31}\right) x+\left(\alpha a_{12}+\beta a_{22}+\gamma a_{32}\right) y+\left(\alpha a_{13}+\beta a_{23}+\gamma a_{33}\right) z\right)\right\|
\end{aligned}
$$

for all $x, y, z \in X$, then $f \equiv 0$.

Now we investigate linearity of a mapping $f: X \rightarrow Y$. The following is a well-known and useful lemma.

Lemma 2.6. Let $f: X \rightarrow Y$ be an additive mapping satisfying $\lim _{t \in \mathbb{R}, t \rightarrow 0} f(t x)=0$ for all $x \in X$. Then $f$ is an $\mathbb{R}$-linear mapping.

Theorem 2.7. Let $f: X \rightarrow Y$ be a nonzero mapping satisfying (2.1) and $\lim _{t \in \mathbb{R}, t \rightarrow 0} f(t x)=0$ for all $x \in X$. Then the following hold:

(a) $f$ is $\mathbb{R}$-linear;

(b) if $\alpha / \beta, \beta / \gamma$ are real numbers, then $a / \alpha=b / \beta=c / \gamma$. 
Proof. (a) For a mapping $f$ satisfying $\lim _{t \in \mathbb{R}, t \rightarrow 0} f(t x)=0$ for all $x \in X$, if we let $x=0$, then we get $f(0)=0$. Since $f$ satisfies (2.1), from (a) in Theorem 2.1 and Lemma 2.6 we conclude that $f$ is $\mathbb{R}$-linear.

(b) Since $f$ is $\mathbb{R}$-linear by (a) and $\alpha / \beta, \beta / \gamma$ are real numbers, by the same reasoning as in the proof of Theorem 2.1(b), we can prove it.

\section{Stability of generalized additive functional inequalities}

In this section, we study the Hyers-Ulam-Rassias stability of generalized additive functional inequalities in Banach spaces.

First of all, we introduce $\alpha$-additivity of a mapping and investigate its properties.

Definition 3.1. For a mapping $f: X \rightarrow Y$, we say that $f$ is $\alpha$-additive if

$$
f(x+\alpha y)=f(x)+\alpha f(y)
$$

for all $x, y \in X$.

Proposition 3.2. If a mapping $f: X \rightarrow Y$ is $\alpha$-additive, then $f$ is additive and $1 / \alpha$-additive.

Proof. Let $f: X \rightarrow Y$ be an $\alpha$-additive mapping. Letting $x=y=0$ in (3.1), we get $f(0)=0$. Letting $x=0$ in (3.1), we get $f(\alpha y)=\alpha f(y)$ for all $y \in X$. Moreover, letting $x=0$ and replacing $y$ by $y / \alpha$ in (3.1), we get $f(y / \alpha)=(1 / \alpha) f(y)$ for all $y \in X$. Hence we obtain

$$
f(x+y)=f\left(x+\alpha \cdot \frac{y}{\alpha}\right)=f(x)+\alpha f\left(\frac{y}{\alpha}\right)=f(x)+f(y)
$$

for all $x, y \in X$ and so $f$ is additive.

On the other hand, we have

$$
f\left(x+\frac{1}{\alpha} y\right)=f\left(\frac{1}{\alpha}(y+\alpha x)\right)=\frac{1}{\alpha} f(y+\alpha x)=f(x)+\frac{1}{\alpha} f(y)
$$

for all $x, y \in X$ and so $f$ is $1 / \alpha$-additive.

Remark 3.3. If a mapping $f: X \rightarrow Y$ is $\alpha$-additive and $\beta$-additive, then we have

$$
f(x+\alpha \beta y)=f(x)+\alpha f(\beta y)=f(x)+\alpha \beta f(y)
$$

for all $x, y \in X$, which implies that $f$ is $\alpha \beta$-additive.

In the following lemma, we give conditions for a mapping $f: X \rightarrow Y$ to be $\mathbb{C}$-linear.

Lemma 3.4. Let $f: X \rightarrow Y$ be an $\alpha$-additive mapping satisfying $\lim _{t \in \mathbb{R}, t \rightarrow 0} f(t x)=0$ for all $x \in X$. If $\alpha$ is not a real number, then $f$ is a $\mathbb{C}$-linear mapping.

Proof. Let $f$ be an $\alpha$-additive mapping satisfying $\lim _{t \in \mathbb{R}, t \rightarrow 0} f(t x)=0$ for all $x \in X$. Since $f$ is additive, by Lemma 2.6, $f$ is $\mathbb{R}$-linear. When $\alpha$ is not real, if we let $\alpha=a+b i$ for some real numbers $a, b(b \neq 0)$, then since $f$ is additive and $\mathbb{R}$-linear, we have

$$
(a+b i) f(x)=f((a+b i) x)=f(a x)+f(b i x)=a f(x)+b f(i x)
$$

and so $f(i x)=i f(x)$ for all $x \in X$, which implies that $f$ is $\mathbb{C}$-linear. 
Jung Rye Lee et al.

Now we are ready to investigate the Hyers-Ulam-Rassias stability of generalized additive functional inequality associated with a linear mapping. Here, we give a lemma for our main result.

Lemma 3.5. Let $f: X \rightarrow Y$ be a mapping. If there exists a function $\psi: X \rightarrow[0, \infty)$ satisfying

$$
\begin{gathered}
\|f(\alpha x)-\alpha f(x)\| \leq \psi(x), \\
\sum_{j=0}^{\infty} \frac{\psi\left(\alpha^{j} x\right)}{|\alpha|^{j}}<\infty
\end{gathered}
$$

for all $x \in X$, then there exists a unique mapping $L: X \rightarrow Y$ satisfying $L(\alpha x)=\alpha L(x)$ and

$$
\|f(x)-L(x)\| \leq \frac{1}{|\alpha|} \sum_{j=0}^{\infty} \frac{\psi\left(\alpha^{j} x\right)}{|\alpha|^{j}}
$$

for all $x \in X$. If, in addition, $f$ is additive, then $L$ is $\alpha$-additive.

Note that this lemma is a special case of the results of [24].

Proof. Replacing $x$ by $\alpha^{j} x$ in (3.6), we get $\left\|f\left(\alpha^{j+1} x\right)-\alpha f\left(\alpha^{j} x\right)\right\| \leq \psi\left(\alpha^{j} x\right)$. Dividing by $|\alpha|^{j+1}$ in the above inequality, we get

$$
\left\|\frac{f\left(\alpha^{j+1} x\right)}{\alpha^{j+1}}-\frac{f\left(\alpha^{j} x\right)}{\alpha^{j}}\right\| \leq \frac{\psi\left(\alpha^{j} x\right)}{|\alpha|^{j+1}}
$$

for all $x \in X$. From the above inequality, we have

$$
\left\|\frac{f\left(\alpha^{n+1} x\right)}{\alpha^{n+1}}-\frac{f\left(\alpha^{q} x\right)}{\alpha^{q}}\right\| \leq \sum_{j=q}^{n}\left\|\frac{f\left(\alpha^{j+1} x\right)}{\alpha^{j+1}}-\frac{f\left(\alpha^{j} x\right)}{\alpha^{j}}\right\| \leq \sum_{j=q}^{n} \frac{1}{|\alpha|} \frac{\psi\left(\alpha^{j} x\right)}{|\alpha|^{j}}
$$

for all $x \in X$ and all nonnegative integers $q$, $n$ with $q<n$. Thus by (3.7), the sequence $\left\{f\left(\alpha^{n} x\right) / \alpha^{n}\right\}$ is Cauchy for all $x \in X$. Since $Y$ is complete, the sequence $\left\{f\left(\alpha^{n} x\right) / \alpha^{n}\right\}$ converges for all $x \in X$. So we can define a mapping $L: X \rightarrow Y$ by

$$
L(x):=\lim _{n \rightarrow \infty} \frac{f\left(\alpha^{n} x\right)}{\alpha^{n}}
$$

for all $x \in X$.

In order to prove that $L$ satisfies (3.8), if we put $q=0$ and let $n \rightarrow \infty$ in the above inequality, then we obtain

$$
\|f(x)-L(x)\| \leq \sum_{j=0}^{\infty} \frac{1}{|\alpha|} \frac{\psi\left(\alpha^{j} x\right)}{|\alpha|^{j}}
$$

for all $x \in X$. 
On the other hand,

$$
L(\alpha x)=\lim _{n \rightarrow \infty} \frac{f\left(\alpha^{n} \alpha x\right)}{\alpha^{n}}=\alpha \lim _{n \rightarrow \infty} \frac{f\left(\alpha^{n+1} x\right)}{\alpha^{n+1}}=\alpha L(x)
$$

for all $x \in X$, as desired.

Now to prove the uniqueness of $L$, let $L^{\prime}: X \rightarrow Y$ be another mapping satisfying $L^{\prime}(\alpha x)=$ $\alpha L^{\prime}(x)$ and (3.8). Then we have

$$
\begin{aligned}
\left\|L(x)-L^{\prime}(x)\right\| & =\frac{1}{|\alpha|^{n}}\left\|L\left(\alpha^{n} x\right)-L^{\prime}\left(\alpha^{n} x\right)\right\| \\
& \leq \frac{1}{|\alpha|^{n}}\left(\left\|L\left(\alpha^{n} x\right)-f\left(\alpha^{n} x\right)\right\|+\left\|L^{\prime}\left(\alpha^{n} x\right)-f\left(\alpha^{n} x\right)\right\|\right) \\
& \leq \frac{2}{|\alpha|^{n}} \cdot \frac{1}{|\alpha|} \sum_{j=0}^{\infty} \frac{\psi\left(\alpha^{j} \alpha^{n} x\right)}{|\alpha|^{j}} \\
& =\frac{2}{|\alpha|} \sum_{j=n}^{\infty} \frac{\psi\left(\alpha^{j} x\right)}{|\alpha|^{j}}
\end{aligned}
$$

which goes to zero as $n \rightarrow \infty$ for all $x \in X$ by (3.7). Consequently, $L$ is a unique desired mapping.

In addition, when $f$ is additive, $L$ is also additive and so the fact of $L(\alpha x)=\alpha L(x)$ for all $x \in X$ gives that $L$ is $\alpha$-additive.

According to Theorem 2.1, the inequality (2.1) can be reduced as the following additive functional inequality

$$
\|\alpha f(x)+\beta f(y)+\gamma f(z)\| \leq\|f(\alpha x+\beta y+\gamma z)\|
$$

for all $x, y, z \in X$.

In the following theorem, we prove the Hyers-Ulam-Rassias stability of the above additive functional inequality.

Theorem 3.6. Let $\xi=-\alpha / \beta$ and let $f: X \rightarrow Y$ be a mapping satisfying $\lim _{t \in \mathbb{R}, t \rightarrow 0} f(t x)=0$ for all $x \in X$. If there exists a function $\varphi: X^{3} \rightarrow[0, \infty)$ satisfying

$$
\begin{gathered}
\|\alpha f(x)+\beta f(y)+\gamma f(z)\| \leq\|f(\alpha x+\beta y+\gamma z)\|+\varphi(x, y, z), \\
\sum_{j=0}^{\infty} \frac{\varphi\left(\xi^{j} x, \xi^{j} y, \xi^{j} z\right)}{\mid \xi^{j}}<\infty, \\
\lim _{t \in \mathbb{R}, t \rightarrow 0} \sum_{j=0}^{\infty} \frac{\varphi\left(\xi^{j} t x, \xi^{j+1} t x, 0\right)}{\mid \xi^{j}}=0
\end{gathered}
$$

for all $x, y, z \in X$, then there exists a unique $\mathbb{R}$-linear and $\xi$-additive mapping $L: X \rightarrow Y$ satisfying

$$
\|f(x)-L(x)\| \leq \frac{1}{|\alpha|} \sum_{j=0}^{\infty} \frac{\varphi\left(\xi^{j} x, \xi^{j+1} x, 0\right)}{|\xi|^{j}}
$$

for all $x \in X$. If, in addition, $\xi$ is not a real number, then $L$ is a $\mathbb{C}$-linear mapping. 
Jung Rye Lee et al.

Proof. Replacing $y=-(\alpha / \beta) x, z=0$ in (3.16), since

$$
\left\|\alpha f(x)+\beta f\left(-\frac{\alpha}{\beta} x\right)\right\| \leq \varphi\left(x,-\frac{\alpha}{\beta} x, 0\right),
$$

we get

$$
\|f(\xi x)-\xi f(x)\| \leq \frac{1}{|\beta|} \varphi(x, \xi x, 0)
$$

for all $x \in X$. If we replace $\psi(x)$ in Lemma 3.5 by $(1 /|\beta|) \varphi(x, \xi x, 0)$, then by (3.17) and Lemma 3.5, there exists a unique mapping $L: X \rightarrow Y$ satisfying $L(\xi x)=\xi L(x)$ for all $x \in X$ and (3.19). In fact, $L(x):=\lim _{n \rightarrow \infty}\left(f\left(\xi^{n} x\right) / \xi^{n}\right)$ for all $x \in X$. Moreover, by $\lim _{t \in \mathbb{R}, t \rightarrow 0} f(t x)=0$ for all $x \in X$ and (3.18), we get

$$
\lim _{t \in \mathbb{R}, t \rightarrow 0}\|L(t x)-f(t x)\| \leq \lim _{t \in \mathbb{R}, t \rightarrow 0} \frac{1}{|\alpha|} \sum_{j=0}^{\infty} \frac{\varphi\left(\xi^{j} t x, \xi^{j+1} t x, 0\right)}{|\xi|^{j}}=0
$$

and so $\lim _{t \in \mathbb{R}, t \rightarrow 0} L(t x)=0$ for all $x \in X$. Since (3.16) and (3.17) give

$$
\begin{aligned}
\|\alpha L(x)+\beta L(y)+\gamma L(z)\| & =\lim _{n \rightarrow \infty}\left\|\frac{\alpha f\left(\xi^{n} x\right)+\beta f\left(\xi^{n} y\right)+\gamma f\left(\xi^{n} z\right)}{\xi^{n}}\right\| \\
& \leq \lim _{n \rightarrow \infty}\left\|\frac{f\left(\xi^{n}(\alpha x+\beta y+\gamma z)\right)}{\xi^{n}}\right\|+\lim _{n \rightarrow \infty} \frac{\varphi\left(\xi^{n} x, \xi^{n} y, \xi^{n} z\right)}{|\xi|^{n}} \\
& =\|L(\alpha x+\beta y+\gamma z)\|+0 \\
& =\|L(\alpha x+\beta y+\gamma z)\|,
\end{aligned}
$$

we conclude that by Theorem 2.1 and Lemma 2.6, a mapping $L$ is $\mathbb{R}$-linear and $\xi$-additive. When $\xi$ is not a real number, by Lemma 3.4, a mapping $L$ is $\mathbb{C}$-linear.

In the above theorem, we remark that when $\xi$ is $-\gamma / \beta$ or $-\alpha / \gamma$, we obtain the same result as in Theorem 3.6.

As an application of Theorem 3.6, we obtain the following stability.

Corollary 3.7. Let $f: X \rightarrow Y$ be a mapping satisfying $\lim _{t \in \mathbb{R}, t \rightarrow 0} f(t x)=0$ for all $x \in X$ and $\xi=-\alpha / \beta$. When $|\alpha|>|\beta|$ and $0<p<1$, or $|\alpha|<|\beta|$ and $p>1$, if there exists $a \geq 0$ satisfying

$$
\|\alpha f(x)+\beta f(y)+\gamma f(z)\| \leq\|f(\alpha x+\beta y+\gamma z)\|+\theta\left(\|x\|^{p}+\|y\|^{p}+\|z\|^{p}\right)
$$

for all $x, y, z \in X$, then there exists a unique $\mathbb{R}$-linear and $\xi$-additive mapping $L: X \rightarrow Y$ satisfying

$$
\|f(x)-L(x)\| \leq \frac{\theta\left(|\alpha|^{p}+|\beta|^{p}\right)}{|\alpha||\beta|\left(|\beta|^{p-1}-|\alpha|^{p-1}\right)}\|x\|^{p}
$$

for all $x \in X$. 
Proof. If we define $\varphi(x, y, z):=\theta\left(\|x\|^{p}+\|y\|^{p}+\|z\|^{p}\right)$, then $\varphi$ satisfies the conditions of (3.17) and (3.18). Thanks to Theorem 3.6, it is proved.

Before closing this section, we establish another stability of generalized additive functional inequalities.

Lemma 3.8. Let $f: X \rightarrow Y$ be a mapping. If there exists a function $\psi: X \rightarrow[0, \infty)$ satisfying (3.6) and

$$
\sum_{j=1}^{\infty}|\alpha|^{j} \psi\left(\frac{x}{\alpha^{j}}\right)<\infty
$$

for all $x \in X$, then there exists a unique mapping $L: X \rightarrow Y$ satisfying $L(\alpha x)=\alpha L(x)$ and

$$
\|f(x)-L(x)\| \leq \frac{1}{|\alpha|} \sum_{j=1}^{\infty}|\alpha|^{j} \psi\left(\frac{x}{\alpha^{j}}\right)
$$

for all $x \in X$. If, in addition, $f$ is additive, then $L$ is $\alpha$-additive.

Note that this lemma is a special case of the results of [24].

Proof. Replacing $x$ by $x / \alpha^{j}$ in (3.6), we get $\left\|f\left(x / \alpha^{j-1}\right)-\alpha f\left(x / \alpha^{j}\right)\right\| \leq \psi\left(x / \alpha^{j}\right)$. Multiplying by $|\alpha|^{j-1}$ in the above inequality, we get

$$
\left\|\alpha^{j-1} f\left(\frac{x}{\alpha^{j-1}}\right)-\alpha^{j} f\left(\frac{x}{\alpha^{j}}\right)\right\| \leq|\alpha|^{j-1} \psi\left(\frac{x}{\alpha^{j}}\right)
$$

for all $x \in X$. From the above inequality, we have

$$
\left\|\alpha^{n} f\left(\frac{x}{\alpha^{n}}\right)-\alpha^{q-1} f\left(\frac{x}{\alpha^{q-1}}\right)\right\| \leq \sum_{j=q}^{n}\left\|\alpha^{j} f\left(\frac{x}{\alpha^{j}}\right)-\alpha^{j-1} f\left(\frac{x}{\alpha^{j-1}}\right)\right\| \leq \sum_{j=q}^{n} \frac{1}{|\alpha|}|\alpha|^{j} \psi\left(\frac{x}{\alpha^{j}}\right)
$$

for all $x \in X$ and all nonnegative integers $q, n$ with $q<n$. Thus by (3.26) the sequence $\left\{\alpha^{n} f\left(x / \alpha^{n}\right)\right\}$ is Cauchy for all $x \in X$. Since $Y$ is complete, the sequence $\left\{\alpha^{n} f\left(x / \alpha^{n}\right)\right\}$ converges for all $x \in X$. So we can define a mapping $L: X \rightarrow Y$ by

$$
L(x):=\lim _{n \rightarrow \infty} \alpha^{n} f\left(\frac{x}{\alpha^{n}}\right)
$$

for all $x \in X$. In order to prove that $L$ satisfies (3.27), if we put $q=1$ and let $n \rightarrow \infty$ in the above inequality, then we obtain

$$
\|f(x)-L(x)\| \leq \frac{1}{|\alpha|} \sum_{j=1}^{\infty}|\alpha|^{j} \varphi\left(\frac{x}{\alpha^{j}}\right)=\frac{1}{|\alpha|} \sum_{j=1}^{\infty}|\alpha|^{j} \psi\left(\frac{x}{\alpha^{j}}\right)
$$

for all $x \in X$. 
Jung Rye Lee et al.

On the other hand,

$$
L(\alpha x)=\lim _{n \rightarrow \infty} \alpha^{n} f\left(\frac{\alpha x}{\alpha^{n}}\right)=\alpha \lim _{n \rightarrow \infty} \alpha^{n-1} f\left(\frac{x}{\alpha^{n-1}}\right)=\alpha L(x)
$$

for all $x \in X$, as desired.

Now to prove the uniqueness of $L$, let $L^{\prime}: X \rightarrow Y$ be another mapping satisfying $L^{\prime}(\alpha x)=$ $\alpha L^{\prime}(x)$ and (3.27). Then we have

$$
\begin{aligned}
\left\|L(x)-L^{\prime}(x)\right\| & =|\alpha|^{n}\left\|L\left(\frac{x}{\alpha^{n}}\right)-L^{\prime}\left(\frac{x}{\alpha^{n}}\right)\right\| \\
& \leq|\alpha|^{n}\left(\left\|L\left(\frac{x}{\alpha^{n}}\right)-f\left(\frac{x}{\alpha^{n}}\right)\right\|+\left\|L^{\prime}\left(\frac{x}{\alpha^{n}}\right)-f\left(\frac{x}{\alpha^{n}}\right)\right\|\right) \\
& \leq 2|\alpha|^{n} \cdot \frac{1}{|\alpha|} \sum_{j=1}^{\infty}|\alpha|^{j} \psi\left(\frac{x}{\alpha^{j} \alpha^{n}}\right) \\
& =\frac{2}{|\alpha|} \sum_{j=1}^{\infty}|\alpha|^{n+j} \psi\left(\frac{x}{\alpha^{n+j}}\right) \\
& =\frac{2}{|\alpha|} \sum_{j=n+1}^{\infty}|\alpha|^{j} \psi\left(\frac{x}{\alpha^{j}}\right)
\end{aligned}
$$

which goes to zero as $n \rightarrow \infty$ for all $x \in X$ by (3.26). Consequently, $L$ is a unique desired mapping.

Theorem 3.9. Let $\xi=-\alpha / \beta$ and let $f: X \rightarrow Y$ be a mapping satisfying $\lim _{t \in \mathbb{R}, t \rightarrow 0} f(t x)=0$ for all $x \in X$. If there exists a function $\varphi: X^{3} \rightarrow[0, \infty)$ satisfying (3.16) and

$$
\begin{gathered}
\sum_{j=1}^{\infty}|\xi|^{j} \varphi\left(\frac{x}{\xi^{j}}, \frac{y}{\xi^{j}}, \frac{z}{\xi^{j}}\right)<\infty, \\
\lim _{t \in \mathbb{R}, t \rightarrow 0} \sum_{j=1}^{\infty}|\xi|^{j} \varphi\left(\frac{t x}{\xi^{j}}, \frac{t x}{\xi^{j-1}}, 0\right)=0
\end{gathered}
$$

for all $x, y, z \in X$, then there exists a unique $\mathbb{R}$-linear and $\xi$-additive mapping $L: X \rightarrow Y$ satisfying

$$
\|f(x)-L(x)\| \leq \frac{1}{|\alpha|} \sum_{j=1}^{\infty}|\xi|^{j} \varphi\left(\frac{x}{\xi^{j}}, \frac{x}{\xi^{j-1}}, 0\right)
$$

for all $x \in X$. If, in addition, $\xi$ is not a real number, then $L$ is a $\mathbb{C}$-linear mapping.

Proof. Replacing $y=-(\alpha / \beta) x, z=0$ in (3.16), we get

$$
\|f(\xi x)-\xi f(x)\| \leq \frac{1}{|\beta|} \varphi(x, \xi x, 0)
$$

for all $x \in X$. Thus by (3.34) and Lemma 3.8, there exists a unique mapping $L: X \rightarrow Y$ satisfying (3.36) and $L(\xi x)=\xi L(x)$ for all $x \in X$. Since $L(x):=\lim _{n \rightarrow \infty} \xi^{n} f\left(x / \xi^{n}\right)$ for all $x \in X$, by $\lim _{t \in \mathbb{R}, t \rightarrow 0} f(t x)=0$ and (3.35), we get

$$
\lim _{t \in \mathbb{R}, t \rightarrow 0}\|L(t x)-f(t x)\| \leq \lim _{t \in \mathbb{R}, t \rightarrow 0} \frac{1}{|\alpha|} \sum_{j=1}^{\infty}|\xi|^{j} \varphi\left(\frac{t x}{\xi^{j}}, \frac{t x}{\xi^{j-1}}, 0\right)=0
$$


and so $\lim _{t \in \mathbb{R}, t \rightarrow 0} L(t x)=0$ for all $x \in X$. It follows from (3.16) and (3.34) that

$$
\begin{aligned}
\|\alpha L(x)+\beta L(y)+\gamma L(z)\| & =\lim _{n \rightarrow \infty}\left\|\xi^{n}\left(\alpha f\left(\frac{x}{\xi^{n}}\right)+\beta f\left(\frac{y}{\xi^{n}}\right)+\gamma f\left(\frac{z}{\xi^{n}}\right)\right)\right\| \\
& \leq \lim _{n \rightarrow \infty}\left\|\xi^{n} f\left(\frac{\alpha x}{\xi^{n}}+\frac{\beta y}{\xi^{n}}+\frac{\gamma z}{\xi^{n}}\right)\right\|+\lim _{n \rightarrow \infty}|\xi|^{n} \varphi\left(\frac{x}{\xi^{n}}, \frac{y}{\xi^{n}}, \frac{z}{\xi^{n}}\right) \\
& =\|L(\alpha x+\beta y+\gamma z)\|+0 \\
& =\|L(\alpha x+\beta y+\gamma z)\|
\end{aligned}
$$

for all $x, y, z \in X$. The rest of the proof is the same as in the corresponding part of the proof of Theorem 3.6.

Corollary 3.10. Let $f: X \rightarrow Y$ be a mapping satisfying $\lim _{t \in \mathbb{R}, t \rightarrow 0} f(t x)=0$ for all $x \in X$. When $|\alpha|>|\beta|$ and $p>1$, or $|\alpha|<|\beta|$ and $0<p<1$, if there exists $a \quad \theta \geq 0$ satisfying

$$
\|\alpha f(x)+\beta f(y)+\gamma f(z)\| \leq\|f(\alpha x+\beta y+\gamma z)\|+\theta\left(\|x\|^{p}+\|y\|^{p}+\|z\|^{p}\right)
$$

for all $x, y, z \in X$, then there exists a unique $\mathbb{R}$-linear and $\xi$-additive mapping $L: X \rightarrow Y$ satisfying

$$
\|f(x)-L(x)\| \leq \frac{\theta\left(|\alpha|^{p}+|\beta|^{p}\right)}{|\alpha||\beta|\left(|\alpha|^{p-1}-|\beta|^{p-1}\right)}\|x\|^{p}
$$

for all $x \in X$.

Proof. If we define $\varphi(x, y, z):=\theta\left(\|x\|^{p}+\|y\|^{p}+\|z\|^{p}\right)$, then $\varphi$ satisfies the conditions of (3.34) and (3.35). Thanks to Theorem 3.9, it is proved.

\section{Acknowledgments}

The first author was supported by Daejin University grants in 2008. The authors would like to thank the referees for a number of valuable suggestions regarding a previous version of this paper.

\section{References}

[1] S. M. Ulam, A Collection of the Mathematical Problems, Interscience Tracts in Pure and Applied Mathematics, no. 8, Interscience, New York, NY, USA, 1960.

[2] D. H. Hyers, "On the stability of the linear functional equation," Proceedings of the National Academy of Sciences of the United States of America, vol. 27, pp. 222-224, 1941.

[3] T. Aoki, "On the stability of the linear transformation in Banach spaces," Journal of the Mathematical Society of Japan, vol. 2, pp. 64-66, 1950.

[4] Th. M. Rassias, "On the stability of the linear mapping in Banach spaces," Proceedings of the American Mathematical Society, vol. 72, no. 2, pp. 297-300, 1978.

[5] P. Găvruţa, "A generalization of the Hyers-Ulam-Rassias stability of approximately additive mappings," Journal of Mathematical Analysis and Applications, vol. 184, no. 3, pp. 431-436, 1994.

[6] Th. M. Rassias, "Problem 16; 2, Report of the 27th International Symposium on Functional Equations," Aequationes Mathematicae, vol. 39, no. 2-3, pp. 292-293, 1990.

[7] Z. Gajda, "On stability of additive mappings," International Journal of Mathematics and Mathematical Sciences, vol. 14, no. 3, pp. 431-434, 1991. 
[8] Th. M. Rassias and P. Šemrl, "On the behavior of mappings which do not satisfy Hyers-Ulam stability," Proceedings of the American Mathematical Society, vol. 114, no. 4, pp. 989-993, 1992.

[9] S.-M. Jung, "On the Hyers-Ulam-Rassias stability of approximately additive mappings," Journal of Mathematical Analysis and Applications, vol. 204, no. 1, pp. 221-226, 1996.

[10] S. Czerwik, Functional Equations and Inequalities in Several Variables, World Scientific, River Edge, NJ, USA, 2002.

[11] D. H. Hyers, G. Isac, and Th. M. Rassias, Stability of Functional Equations in Several Variables, vol. 34 of Progress in Nonlinear Differential Equations and Their Applications, Birkhäuser, Boston, Mass, USA, 1998.

[12] D. G. Bourgin, "Classes of transformations and bordering transformations," Bulletin of the American Mathematical Society, vol. 57, pp. 223-237, 1951.

[13] Th. M. Rassias, "The problem of S. M. Ulam for approximately multiplicative mappings," Journal of Mathematical Analysis and Applications, vol. 246, no. 2, pp. 352-378, 2000.

[14] Th. M. Rassias, "On the stability of functional equations in Banach spaces," Journal of Mathematical Analysis and Applications, vol. 251, no. 1, pp. 264-284, 2000.

[15] Th. M. Rassias, "On the stability of functional equations and a problem of Ulam," Acta Applicandae Mathematicae, vol. 62, no. 1, pp. 23-130, 2000.

[16] Th. M. Rassias, Functional Equations, Inequalities and Applications, Kluwer Academic Publishers, Dordrecht, The Netherlands, 2003.

[17] F. Skof, "Local properties and approximation of operators," Rendiconti del Seminario Matematico e Fisico di Milano, vol. 53, pp. 113-129, 1983.

[18] A. Gilányi, "Eine zur Parallelogrammgleichung äquivalente Ungleichung," Aequationes Mathematicae, vol. 62, no. 3, pp. 303-309, 2001.

[19] J. Rätz, "On inequalities associated with the Jordan-von Neumann functional equation," Aequationes Mathematicae, vol. 66, no. 1-2, pp. 191-200, 2003.

[20] W. Fechner, "Stability of a functional inequality associated with the Jordan-von Neumann functional equation," Aequationes Mathematicae, vol. 71, no. 1-2, pp. 149-161, 2006.

[21] A. Gilányi, "On a problem by K. Nikodem," Mathematical Inequalities \& Applications, vol. 5, no. 4, pp. 707-710, 2002.

[22] C. Park, Y. S. Cho, and M.-H. Han, "Functional inequalities associated with Jordan-von Neumanntype additive functional equations," Journal of Inequalities and Applications, vol. 2007, Article ID 41820, 13 pages, 2007.

[23] Y.-S. Cho and H.-M. Kim, "Stability of functional inequalities with Cauchy-Jensen additive mappings," Abstract and Applied Analysis, vol. 2007, Article ID 89180, 13 pages, 2007.

[24] G. L. Forti, "Comments on the core of the direct method for proving Hyers-Ulam stability of functional equations," Journal of Mathematical Analysis and Applications, vol. 295, no. 1, pp. 127-133, 2004. 